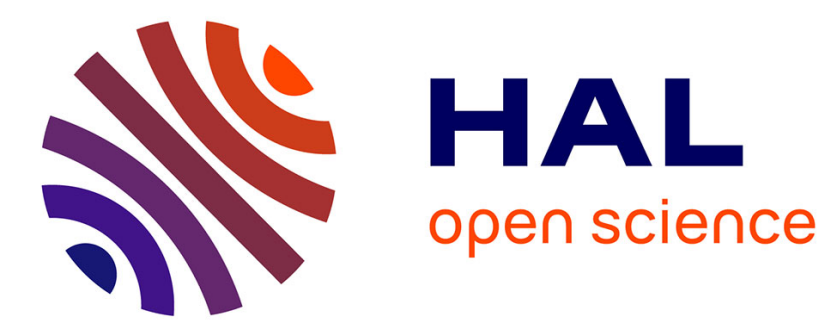

\title{
Nonlinear Filtering with Transfer Operator
}

\author{
Parikshit Dutta, Abhishek Halder, Raktim Bhattacharya
}

\section{To cite this version:}

Parikshit Dutta, Abhishek Halder, Raktim Bhattacharya. Nonlinear Filtering with Transfer Operator. IEEE American Control Conference, Jun 2013, Washington, D.C., United States. pp.3069-3074, 10.1109/ACC.2013.6580302 . hal-00785159

\section{HAL Id: hal-00785159 \\ https://hal.inria.fr/hal-00785159}

Submitted on 5 Feb 2013

HAL is a multi-disciplinary open access archive for the deposit and dissemination of scientific research documents, whether they are published or not. The documents may come from teaching and research institutions in France or abroad, or from public or private research centers.
L'archive ouverte pluridisciplinaire HAL, est destinée au dépôt et à la diffusion de documents scientifiques de niveau recherche, publiés ou non, émanant des établissements d'enseignement et de recherche français ou étrangers, des laboratoires publics ou privés. 


\title{
Nonlinear Filtering with Transfer Operator
}

\author{
Parikshit Dutta, Abhishek Halder, and Raktim Bhattacharya
}

\begin{abstract}
This paper presents a new nonlinear filtering algorithm that is shown to outperform state-of-the-art particle filters with resampling. Starting from the Itô stochastic differential equation, the proposed algorithm harnesses KarhunenLoéve expansion to derive an approximate non-autonomous dynamical system, for which transfer operator based density computation can be performed in exact arithmetic. It is proved that the algorithm is asymptotically consistent in mean-square sense. Numerical results demonstrate that explicitly accounting prior dynamics entail significant performance improvement for nonlinear non-Gaussian estimation problems with infrequent measurement updates, as compared to the performance of particle filters.
\end{abstract}

\section{INTRODUCTION}

On a probability space $(\Omega, \mathscr{F}, \mathbb{P})$ with filtration $\left\{\mathscr{F}_{t}\right\}_{t \geqslant 0}$, consider the nonlinear estimation problem associated with the Itô stochastic differential equations (SDEs)

$$
\begin{aligned}
& d x(t)=f(x(t), t, \delta) d t+d \mathcal{W}(\omega, t), \\
& d y(t)=h(x(t), t, \delta) d t+d \mathcal{V}(\omega, t),
\end{aligned}
$$

where at time instance $t$, the state vector $x(t) \in \mathbb{R}^{n}$, and the measurement vector $y(t) \in \mathbb{R}^{m} . \delta \in \mathbb{R}^{p}$ is the parameter vector, and $\mathcal{W}(\omega, t): \Omega \times \mathbb{R}^{+} \mapsto \mathbb{R}^{n}, \mathcal{V}(\omega, t): \Omega \times \mathbb{R}^{+} \mapsto$ $\mathbb{R}^{m}$ are mutually independent Wiener processes denoting process and measurement noise, respectively. Further, $\omega \in \Omega$, and the functions $f($.$) and h($.$) represent the dynamics and$ measurement models, respectively.

State and parameter estimation for nonlinear systems such as above, are commonly done using sequential Monte Carlo (SMC) methods, particle filter being the most popular amongst them [1]. These algorithms follow traditional prediction-update framework where the prior is predicted using state dynamics, followed by a Bayesian update using measurement model, resulting the posterior. It is well known [2] that these methods require large number of samples for convergence, leading to higher computational cost. This problem is usually tackled by combining particle filters with resampling [3], [4], commonly known as bootstrap filters [5]. However, resampling may introduce loss of diversity amongst particles [6]. Several other methods like regularized particle filter [7], and filters with Markov Chain Monte Carlo $(M C M C)$ move step [8], have been proposed to enhance sample diversity. At the same time, even with resampling,

Parikshit Dutta is with INRIA Rhône Alpes \& Laboratoire Jean Kuntzmann, 655 avenue de l'Europe, 38330 Montbonnot, France, parikshit.dutta@inria.fr; Abhishek Halder and Raktim Bhattacharya are with the Department of Aerospace Engineering, Texas A\&M University, College Station, TX 77843, USA, \{ahalder, raktim\}etamu.edu. This research was supported by NSF award \#1016299 with Helen Gill as the Program Manager. due to the simulation based nature of these filters, the sample size scales exponentially with state dimension [9]. To circumvent this problem, particle filters based on RaoBlackwellization [10] have been proposed to partially solve the estimation problem analytically. However, its application remains limited to systems where the required partition of the state space is possible.

The main idea of this paper is to recognize the fact that much of the computational burden of particle filter, stems from the Monte Carlo approximation of the prior. Lack of statistically consistent methods for high dimensional uncertainty propagation, has stymied the accurate computation of prior density. In the previous work, the authors developed [11] Perron-Frobenius (PF) operator [12] based methods for numerically efficient uncertainty propagation schemes for nonlinear systems with parametric and initial condition uncertainties. This was achieved by solving the characteristic ordinary differential equation (ODE) corresponding to the Liouville partial differential equation (PDE), along the trajectories in the extended state space $z:=\left[\begin{array}{ll}x & \delta\end{array}\right]^{\top}$. In the estimation setting, it was observed [13] that prior probability density function (PDF) computed via PF operator, followed by Bayesian update, outperformed particle filter in the absence of process noise.

In the presence of process noise, the transport PDE associated with forward Kolmogorov operator is the FokkerPlanck-Kolmogorov (FPK) equation [14], which being a second order PDE, does not enjoy method-of-characteristics (MOC) based ODE formulation. Function approximation techniques for solving FPK eqn. usually suffer from the "curse of dimensionality" [15]. This severely limits the accuracy of prior computation, and hence that of the nonlinear filters. An alternative approach was proposed recently by the authors [16], where instead of directly approximating the prior, the process noise was approximated by a finite-term Karhunen-Loéve (KL) expansion resulting an approximate state dynamics. Next, the MOC based PF operator computation was performed on this approximate non-autonomous dynamical system in exact arithmetic. [16] provided strong numerical evidence that such two step "first $K L$, then $P F$ " (henceforth $K L P F$ ) algorithm is asymptotically consistent in distribution. However, two issues remained unsettled.

1) In [16], the distributional consistency was algorithmically verified. A rigorous proof for convergence was lacking. Also, it was not clear whether the distributional convergence is only sufficient, i.e. whether a stronger notion of convergence holds true.

2) No detailed numerical investigation was performed to assess the filtering performance improvement resulting 
from KLPF algorithm, vis-a-vis with particle filter.

This paper has two key contributions. First, we prove that solution of the KL approximated dynamics, converges to that of the true Itô SDE in mean-square (m.s.) sense. This is indeed stronger than the distributional convergence of [16]. Further, the m.s. convergence is shown to be necessary and sufficient. Second, we provide strong numerical results showing that the proposed algorithm, henceforth referred as $K L P F$ filter, achieves superior estimation accuracy than the particle filter with resampling.

The rest of this paper is structured as follows. Section II describes the KLPF formulation for computing prior PDF and provides m.s. convergence guarantees. The nonlinear filtering algorithm is introduced in Section III. Section IV contains numerical results for both linear Gaussian and nonlinear non-Gaussian estimation problems. Section V concludes the paper.

\section{Notation}

$\mathcal{I}_{n}$ denotes the $n$-by- $n$ identity matrix, and diag $(\cdot)$ denotes the diagonal matrix. The symbol $\mathcal{N}(\mu, \Sigma)$ denotes joint Gaussian PDF with mean $\mu$ and covariance $\Sigma$. $\mathbb{N}$ stands for the set of natural numbers, $\operatorname{tr}(\cdot)$ denotes the trace of a matrix, and $\operatorname{div}(\cdot)$ denotes the divergence operator. The symbol $\delta_{i j}$ represents Kronecker delta, and the symbol $\wedge$ denotes minimum.

\section{ApPROXIMATING PRIOR DYNAMiCS}

\section{A. KLPF Formulation}

Given the Itô SDE (1), we write an approximate dynamical system corresponding to its Langevin ODE form for the $j^{\text {th }}$ state:

$$
\dot{x}_{N}^{(j)}=f^{(j)}\left(x_{N}(t), t, \delta\right)+\sum_{i=1}^{N} \sqrt{\Lambda_{i}} \zeta_{i}^{(j)}(\omega) e_{i}(t),
$$

where $j=1,2, \ldots, n$. Further, $\left\{\Lambda_{i}, e_{i}(t)\right\}_{i=1}^{\infty}$ is the sequence of eigenvalue-eigenfunction pairs of the covariance function $C\left(t_{1}, t_{2}\right)$ associated with the additive stationary process noise, and $\zeta_{i}(\omega)$ are i.i.d. random variables drawn from the distribution of the noise stochastic process. For example, if $\mathcal{W}(\omega, t)$ is Wiener process with $C\left(t_{1}, t_{2}\right)=$ $\sigma^{2}\left(t_{1} \wedge t_{2}\right), t_{1}, t_{2} \in[0, T]$, then $\left\{\Lambda_{i}, e_{i}(t)\right\}_{i=1}^{\infty}$ is the eigenpair sequence for Gaussian white noise $\eta(\omega, t)$, and $\zeta_{i}(\omega) \sim$ $\mathcal{N}\left(0, \sigma^{2}\right)$. In effect, the second term in the RHS of (3), is the $N$-term KL expansion for $\eta(\omega, t)$. We affix subscript $N$ to the states $\left(x_{N}(t)\right)$ of the approximate dynamics $(3)$, to distinguish them from the sample paths $(x(t))$ of the original SDE (1).

Next, we augment (3) with the characteristic ODE

$$
\dot{\varphi^{-}}=-\operatorname{div}(\tilde{f}) \varphi^{-}
$$

where $\tilde{f}$ denotes the RHS nonlinearity of (3), and $\varphi^{-}\left(x_{N}(t), t, \delta\right)$ denotes the prior at time $t$, supported over the extended state space $\left[\begin{array}{ll}x_{N} & \delta\end{array}\right]^{\top} \in \mathbb{R}^{n+p}$. Consequently, (4) computes the evolution of joint prior PDF along the characteristic curves $x_{N}(t)$. Notice that we do not assume
Stochastic evolution equation

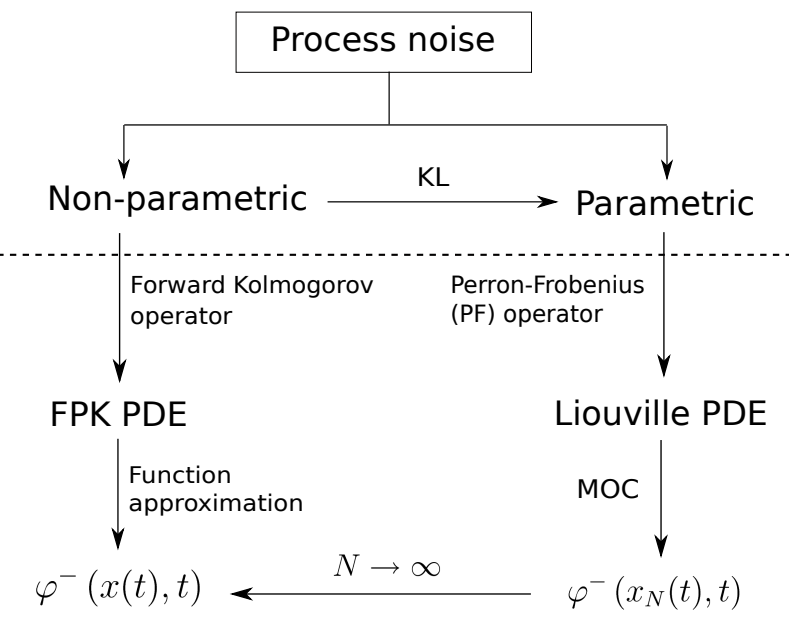

Fig. 1. Summary of the KLPF formulation.

the process noise to be Gaussian. As long as the additive noise has finite second moment, we can write down the approximate dynamical system (3) via the noise KL expansion (Table I). The overall formulation is summarized in Fig. 1 .

It is well-known [18] that as $N \rightarrow \infty$, the finite-term noise KL expansion $\sum_{i=1}^{N} \sqrt{\Lambda_{i}} \zeta_{i}^{(j)}(\omega) e_{i}(t)$ converges uniformly to the unstructured noise $\eta(\omega, t)$ in m.s. sense. However, to justify our formulation, it remains to answer whether $x_{N}(t)$ converges to that of $x(t)$, and in what sense. The following sub-section answers this issue. For notational ease, we disregard uncertainty on parameter $\delta$, without loss of generality. It is straightforward to verify that the following results generalize to extended state space.

\section{B. Quality of Approximation}

1) Asymptotic convergence: In [16], it was verified through simulation that as $N \rightarrow \infty, x_{N}(t) \rightarrow x(t)$ in distribution, i.e. $\varphi^{-}\left(x_{N}(t), t\right) \rightarrow \varphi^{-}(x(t), t), \forall t \geqslant 0$. Here we prove the stronger result that $x_{N}(t) \rightarrow x(t)$ in m.s. sense. All proofs are given in the Appendix.

Theorem 1: Let $x(\omega, t)$ be the solution of the nonlinear Itô SDE

$$
\begin{aligned}
d x(t) & =f(x(t), t) d t+d \mathcal{W}(\omega, t), \\
\Rightarrow \frac{d}{d t} x(t) & =f(x, t)+\eta(\omega, t),
\end{aligned}
$$

where $d W(\omega, t)=\eta(\omega, t) d t$, and $f: \mathbb{R}^{n} \times[0, T] \rightarrow \mathbb{R}^{n}$ satisfies the following:

1) non-explosion condition: $\exists D \geq 0$, s.t. $|f(x, t)|<$ $D(1+|x|)$ where $x \in \mathbb{R}^{n}, t \in[0, T]$;

2) Lipschitz condition: $\exists C \longrightarrow 0$, s.t. $|f(x, t)-f(\breve{x}, t)|<C|x-\breve{x}|$, where $x, \breve{x} \in \mathbb{R}^{n}$, $t \in[0, T]$.

Let $x_{N}(t)$ be solution of the ODE

$$
\frac{d}{d t} x_{N}(t)=f\left(x_{N}(t), t\right)+\eta_{N}(\omega, t),
$$


TABLE I

NoISE KL EXPANSION: EXAMPLES

\begin{tabular}{|l|l|l|l|}
\hline Noise $\mathcal{W}(\omega, t)$ in SDE & $C\left(t_{1}, t_{2}\right)$ for $\mathcal{W}(\omega, t)$ & White noise $\eta(\omega, t)$ in Langevin ODE & KL expansion of $\eta(\omega, t), 0<t \leqslant T$ \\
\hline \hline Wiener process & $\sigma^{2}\left(t_{1} \wedge t_{2}\right)$ & Gaussian white noise & $\sqrt{\frac{2}{T} \sum_{i=1}^{\infty} \zeta_{i}(\omega) \cos \left(\left(i-\frac{1}{2}\right) \frac{\pi t}{T}\right)}$ \\
\hline Compound Poisson process & $\lambda \sigma^{2}\left(t_{1} \wedge t_{2}\right)+(\lambda \mu)^{2} t_{1} t_{2}$ & Poisson white noise (Appendix A) & $\sum_{i=1}^{\infty} \bar{\zeta}_{i}(\omega) \frac{\frac{2}{\beta_{i}} \sqrt{\Lambda_{i}}}{\sqrt{2 T-\beta_{i} \sin \frac{2 T}{\beta_{i}}}} \cos \left(\frac{t}{\beta_{i}}\right)$ \\
\hline
\end{tabular}

where $\eta_{N}(\omega, t)$ is the $N$-term truncated orthonormal expansion of $\eta(\omega, t)$, and $\mathbb{E}\left[\int_{0}^{T} \eta_{N}(\omega, t) d t\right]<\infty$. Then,

$$
\lim _{N \rightarrow \infty} \mathbb{E}\left|x(t)-x_{N}(t)\right|^{2}=0,
$$

iff $x_{N}(t)$ is the KL expansion of $x(t)$.

Theorem 1 states conditions upon the solutions of approximated and true systems for m.s. convergence to hold, under certain assumptions on the nonlinearities. No condition has been imposed yet on the initial states, which we investigate next.

Theorem 2: Given the stochastic dynamical system

$$
d x(t)=f(x(t), t) d t+d \mathcal{W}(\omega, t)
$$

and its corresponding $N$-term KL approximation given by

$$
d x_{N}^{(j)}(t)=f^{(j)}\left(x_{N}(t), t\right) d t+\sum_{i=1}^{N} \sqrt{\Lambda_{i}} \zeta_{i}^{(j)}(\omega) e_{i}(t) d t
$$

where, $\lim _{N \rightarrow \infty} \mathbb{E}\left|\mathcal{W}^{(j)}(\omega, t)-\sum_{i=1}^{N} \sqrt{\Lambda_{i}} \zeta_{i}^{(j)}(\omega) e_{i}(t)\right|^{2}=0$, $\forall j=1,2, \ldots, n$. Then, $\lim _{N \rightarrow \infty} \mathbb{E}\left|x(t)-x_{N}(t)\right|^{2}=0$, if $x(0)=x_{N}(0)$.

Corollary 3: Suppose $x_{N}(0) \neq x(0)$. If $x_{N}(0)$ is the generalized polynomial chaos ( $\mathrm{gPC}$ ) expansion of $x(0)$, then $\lim _{N \rightarrow \infty} \mathbb{E}\left|x(t)-x_{N}(t)\right|^{2}=0$.

\section{KLPF FILTER}

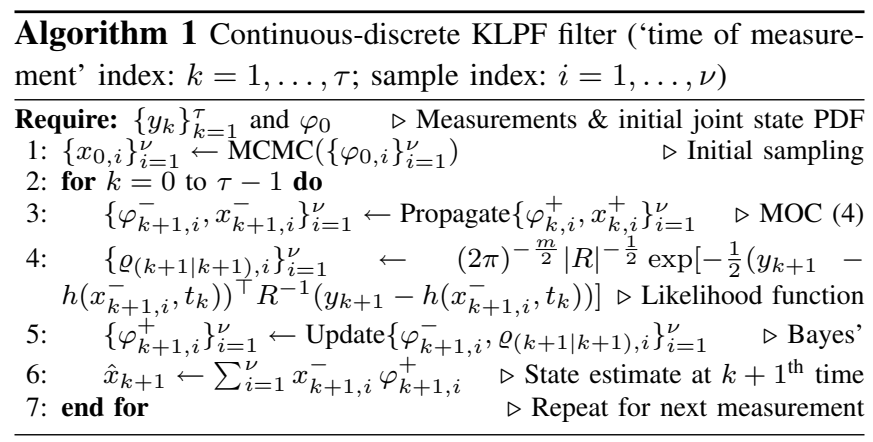

\section{NumericAl RESUlts}

In this subsection, we consider two examples for which the estimation problem is exactly solvable and hence the true posterior is known. To demonstrate the performance improvement achieved by KLPF compared to particle filter, we must show that the KLPF posterior is closer to the true posterior, than particle filter. In other words, the "distance" between KLPF posterior and true posterior, must remain smaller than the "distance" between particle filter posterior and true posterior, for all times. The notion of distributional distance used here, is the quadratic Wasserstein metric of order two (denoted as ${ }_{2} W_{2}$ ), that measures the difference in shapes between the two statistical distributions under comparison.

Definition 1: (Wasserstein distance) Consider a metric space $\left(M, \ell_{p}\right)$ and let $x, \widetilde{x} \in M$. For $q \in \mathbb{N}$, let $\mathcal{P}_{q}(M)$ denote the collection of all probability measures $\mu$ supported on $M$, which have finite $q^{\text {th }}$ moment. Then the $\ell_{p}$ Wasserstein distance of order $q$, denoted as ${ }_{p} W_{q}$, between two probability measures $\varsigma_{1}, \varsigma_{2} \in \mathcal{P}_{q}(M)$, is defined as

${ }_{p} W_{q}\left(\varsigma_{1}, \varsigma_{2}\right):=\left(\inf _{\mu \in \mathcal{M}\left(\varsigma_{1}, \varsigma_{2}\right)} \int_{M \times M}\|x-\widetilde{x}\|_{\ell_{p}}^{q} d \mu(x, \widetilde{x})\right)^{\frac{1}{q}}$ where $\mathcal{M}\left(\varsigma_{1}, \varsigma_{2}\right)$ is the set of all measures supported on the product space $M \times M$, with first marginal $\varsigma_{1}$ and second marginal $\varsigma_{2}$.

Remark 1: Intuitively, Wasserstein distance quantifies the minimum amount of work required to convert one distributional shape to the other, and can be interpreted as the cost for Monge-Kantorovich optimal transportation plan [19]. We set $p=q=2$ (see [20] for details) for comparing posteriors, and for notational ease, henceforth denote ${ }_{2} W_{2}$ as $W$. For absolutely continuous measures $\varsigma_{1}$ and $\varsigma_{2}$, with PDFs $\varphi_{1}$ and $\varphi_{2}$, we can write $W\left(\varphi_{1}, \varphi_{2}\right)$ in lieu of $W\left(\varsigma_{1}, \varsigma_{2}\right)$.

Remark 2: For multivariate Gaussians, $W$ admits [21] a closed form expression, given by

$$
\begin{aligned}
W\left(\mathcal{N}\left(\mu_{1}, \Sigma_{1}\right), \mathcal{N}\left(\mu_{2}, \Sigma_{2}\right)\right) & =\left(\left\|\mu_{1}-\mu_{2}\right\|_{2}^{2}+\operatorname{tr}\left(\Sigma_{1}+\Sigma_{2}\right)\right. \\
& \left.-2 \operatorname{tr}\left[\sqrt{\Sigma_{1}} \Sigma_{2} \sqrt{\Sigma_{1}}\right]^{1 / 2}\right)^{1 / 2} .
\end{aligned}
$$

In general, computation of $W$ from definition 1 necessitates solving a linear program (LP). We refer the readers to [22] for details of this computation. 


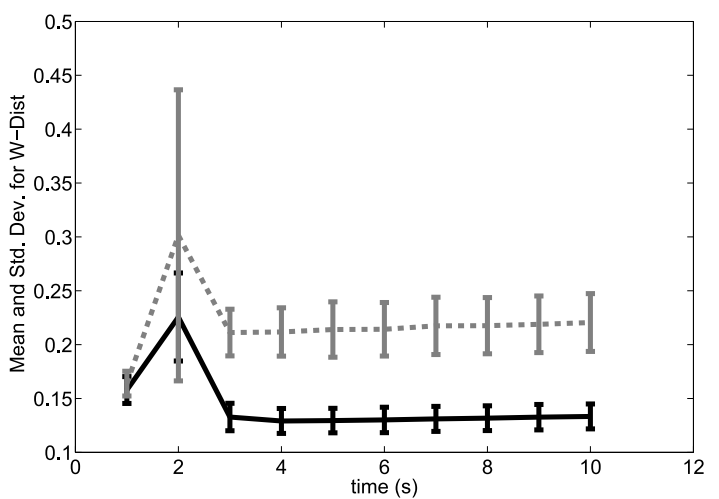

Fig. 2. Plot of means and standard deviations of the Wasserstein distances of the posteriors from KLPF filter (solid line) and the particle filter (hyphenated line) for the Kalman filter. The vertical lines about the means represent $\pm 1 \sigma$ limits.

\section{A. Kalman filter}

Let us consider the continuous-discrete Kalman filter with continuous-time state dynamics

$$
\dot{x}(t)=-0.05 \mathcal{I}_{2} x(t)+\left[\begin{array}{ll}
1 & 1
\end{array}\right]^{\top} \eta(t),
$$

and discrete-time measurement model

$$
y_{k}=\left[\begin{array}{ll}
1 & 1
\end{array}\right] x_{k}+v_{k}, \quad k \in \mathbb{N},
$$

where $\eta(t)$ and $v_{k}$ are independent zero mean Gaussian white noise processes, with variances $Q=1 / 8$ and $R=1 / 4$, respectively. We assume the initial joint state PDF to be $\mathcal{N}\left(\left[\begin{array}{ll}1 & 1\end{array}\right]^{\top}, \operatorname{diag}(1,1)\right)$.

From this initial state PDF, we draw 100 sample sets, each with sample size 500. Then using (10), we compute two Wasserstein time histories: $W\left(\varphi_{\text {Kalman }}^{+}(t), \varphi_{\text {Particle }}^{+}(t)\right)$ and $W\left(\varphi_{\text {Kalman }}^{+}(t), \varphi_{\mathrm{KLPF}}^{+}(t)\right)$, where $\varphi_{\text {Kalman }}^{+}(t), \varphi_{\text {Particle }}^{+}(t)$ and $\varphi_{\mathrm{KLPF}}^{+}(t)$ denote posteriors at time $t$, obtained from Kalman filter, particle filter and KLPF filter, respectively. The means and standard deviations of these time histories are shown in Fig. 2 This plot shows that the KLPF filter posterior remains indeed closer to the Kalman posterior, compared to the particle filter posterior.

\section{B. Beneš filter}

Benes filter is one of the few [23] nonlinear filters which admit a known finite-dimensional solution of the nonlinear estimation problem. Here, the nonlinear drift in state dynamics, is assumed to satisfy a Riccati differential equation [24] and the measurement model is taken to be affine in states. We consider the continuous-continuous scalar Beneš filtering problem of the form:

$$
\begin{aligned}
d x(t) & =\frac{\kappa e^{x}-e^{-x}}{\kappa e^{x}+e^{-x}} d t+d \mathcal{W}(\omega, t), \\
d y(t) & =x(t) d t+d \mathcal{V}(\omega, t),
\end{aligned}
$$

with $\kappa=0.5$ and deterministic initial condition $x_{0}$. The process and measurement noise densities are $\mathcal{N}(0, Q)$ and $\mathcal{N}(0, R)$ respectively, with $Q=1, R=10$. It can be shown [25] that the drift nonlinearity satisfies the necessary Riccati

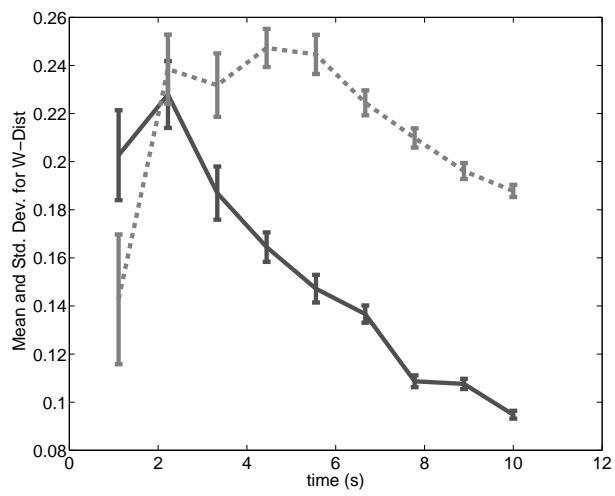

Fig. 3. Plot of means and standard deviations of the Wasserstein distances of the posteriors from KLPF filter (solid line) and the particle filter (hyphenated line) for the Bene $\breve{s}$ filter. The vertical lines about the means represent $\pm 1 \sigma$ limits.

condition and the resulting solution [26] is given by the normalized posterior density

$$
\begin{gathered}
\varphi\left(x(t) \mid \mathcal{Y}_{t}\right)=\sqrt{\frac{\operatorname{coth}(t)}{2 \pi}}\left(\frac{\kappa e^{x}+e^{-x}}{\kappa e^{I_{t}(y(\omega))}+e^{-I_{t}(y(\omega))}}\right) \\
\exp \left(-\frac{1}{2} \Gamma(t)\right),
\end{gathered}
$$

where $\mathcal{Y}_{t}$ is the history (filtration) till time $t$, and

$$
\begin{aligned}
I_{t}(y(\omega)) & :=\operatorname{sech}(t)\left[x_{0}+\int_{0}^{t} \sinh (s) d y_{s}(\omega)\right],(16) \\
\Gamma(t) & :=\tanh (t)+\operatorname{coth}(t)\left(x-I_{t}(y(\omega))\right)^{2}
\end{aligned}
$$

Notice that for this nonlinear non-Gaussian estimation problem, unlike Kalman filter case, we can not write the Wasserstein distance between the true posterior (15) and particle filter/KLPF posterior, as an analytical expression in terms of the respective sufficient statistics. Thus, in order to compute the Wasserstein time history, we resort to the LP formulation [22]. At each time, we sample (15) using the MetropolisHastings MCMC technique [27], and solve the LP between the sampled true Beneš posterior and particle filter/KLPF posterior, to result the normalized Wasserstein trajectories shown in Fig. 3. Like the Kalman filter case, as time progresses, KLPF posterior gets closer, compared to particle filter, to true Beneš posterior.

\section{CONCLUSIONS}

A new nonlinear filtering algorithm is presented in this paper, that is shown to outperform the estimation accuracy of particle filters in numerical simulations. This is achieved by explicitly taking the prior dynamics into account. Contrary to the traditional "top-down" approach of numerically solving the FPK PDE via function approximation, a "bottom up" approach for prior computation is developed by first approximating the problem via spectral parametrization of the noise, and then solving that approximate problem in exact arithmetic via MOC computation of the transfer operator. The resulting algorithm, dubbed as KLPF filter, is a nonparticle filter [29], and is amenable to both Gaussian and non-Gaussian process noise. 


\section{APPENDIX}

\section{A. KL Expansion of Poisson White Noise}

We first obtain the KL expansion of compound Poisson process [17] with covariance kernel $C(s, t)=\lambda \sigma^{2}(s \wedge t)+$ $s t(\lambda \mu)^{2}$, as listed in Table I This requires us to solve the associated Fredholm integral equation of second kind, given by $\Lambda_{n} e_{n}(s)=(\lambda \mu)^{2} s \int_{0}^{T} t e_{n}(t) d t+\lambda \sigma^{2} \int_{0}^{s} t e_{n}(t) d t+$ $\lambda \sigma^{2} s \int_{s}^{T} e_{n}(t) d t$, which, upon differentiating w.r.t. $s$, yields

$$
\Lambda_{n} e_{n}^{\prime}(s)=(\lambda \mu)^{2} \int_{0}^{T} t e_{n}(t) d t+\lambda \sigma^{2} \int_{s}^{T} e_{n}(t) d t, \Lambda_{n} .
$$

Thus, we have $e_{n}^{\prime \prime}(s)=-\lambda \sigma^{2} e_{n}(s)$, which solves as $e_{n}(t)=A \sin \left(\frac{t}{\beta_{n}}\right)+B \cos \left(\frac{t}{\beta_{n}}\right)$, where $\beta_{n} \triangleq \sqrt{\frac{\Lambda_{n}}{\lambda \sigma^{2}}}$. Imposing boundary conditions $\Lambda_{n} e_{n}(0)=0 \Rightarrow B=0$, and $\int_{0}^{T} e_{n}^{2}(t) d t=1 \Rightarrow A=\frac{2}{\sqrt{\left[2 T-\beta_{n} \sin \frac{2 T}{\beta_{n}}\right]}}$. Hence, the eigenfunction for Poisson process for $t \in[0, T]$, is $e_{n}(t)=\frac{2}{\sqrt{\left[2 T-\beta_{n} \sin \frac{2 T}{\beta_{n}}\right]}} \sin \left(\frac{t}{\beta_{n}}\right)$.

Now we substitute $s=T$, and $e_{n}(t)$ as derived above, in the Fredholm integral equation, to obtain

$$
\Lambda_{n} \sin \frac{T}{\beta_{n}}=\left[(\lambda \mu)^{2} T+\lambda \sigma^{2}\right]\left[\beta_{n}^{2} \sin \frac{T}{\beta_{n}}-T \beta_{n} \cos \frac{T}{\beta_{n}}\right] .
$$

Similarly, substituting $s=T$, and $e_{n}(t)$ in $(18)$ results

$$
\frac{\Lambda_{n}}{\beta_{n}} \cos \frac{T}{\beta_{n}}=(\lambda \mu)^{2} \beta_{n}^{2} \sin \frac{T}{\beta_{n}}-(\lambda \mu)^{2} T \beta_{n} \cos \frac{T}{\beta_{n}} .
$$

From (19) and 20, we have

$$
\tan \left(\sigma T \sqrt{\frac{\lambda}{\Lambda_{n}}}\right)=\left[1+\frac{1}{\lambda T}\left(\frac{\sigma}{\mu}\right)^{2}\right]\left(\sigma T \sqrt{\frac{\lambda}{\Lambda_{n}}}\right),
$$

where $\lambda, \sigma, \mu, T>0 ; n \in \mathbb{N}$. Consequently, the $\mathrm{KL}$ expansion of compound Poisson process $Y(\omega, t)$ is given by $Y(\omega, t)=\sum_{n=1}^{\infty} \sqrt{\Lambda_{n}} \bar{\zeta}_{n}(\omega) e_{n}(t)$, where $\bar{\zeta}_{i}(\omega) \sim \mathcal{N}(0,1)$ and $\Lambda_{n}$ solves the transcendental equation (21). Next, we take the formal derivative (in m.s. sense) of the KL expansion of $Y(\omega, t)$, to arrive at the KL expansion of Poisson white noise, given in the second row, right-most column in Table 【.

\section{B. Proof for Theorem 1}

$(\Leftarrow)$ Given (7) holds, we need to show $x_{N}(\omega, t)$ is the KL expansion of $x(\omega, t)$. Let $\left\{\psi_{m}(t)\right\}_{m=1}^{\infty}$ be any orthonormal basis. Then $x(\omega, t)$ can be written as a convergent sum in $L_{2}(\Omega, \mathscr{F}, \mathbb{P})$, i.e. $x(\omega, t)=\sum_{m=1}^{\infty} b_{m} c_{m}(\omega) \psi_{m}(t)$.

Let $x_{N}(\omega, t)$ be an $N$-term m.s. convergent approximation of $x(\omega, t)$, and the resulting truncation error equals $\mathscr{E}_{N}(\omega, t)=\sum_{m=N+1}^{\infty} b_{m} c_{m}(\omega) \psi_{m}(t)$. Further, projecting $x(\omega, t)$ onto the basis $\psi_{m}(t)$ results $c_{m}(\omega)=$ $\frac{1}{b_{m}} \int_{0}^{T} x(\omega, t) \psi_{m}(t) d t$. For convergence, the basis $\psi_{m}(t)$ should minimize $\int_{0}^{T} \mathbb{E}\left[\mathscr{E}_{N}(\omega, t)\right] d t$ subject to the orthonormality constraint $\int_{0}^{T} \phi_{m}(t) \phi_{k}(t) d t=\delta_{m k}, \forall m, k \in \mathbb{N}$.
Introducing $b_{m}^{2}$ as Lagrange multipliers and using the above derived formula for $c_{m}(\omega)$, the first order optimality condition yields $\int_{0}^{T} C_{x x}\left(t_{1}, t_{2}\right) \psi_{m}\left(t_{1}\right) d t_{1}=b_{m}^{2} \psi_{m}\left(t_{2}\right)$, which is the Fredholm integral equation of second kind for the covariance function of random process $x(\omega, t)$. Hence $\left\{b_{m}^{2}, \psi_{m}(t)\right\}_{m=1}^{\infty}$ is the eigenvalue-eigenfunction sequence for $C_{x x}\left(t_{1}, t_{2}\right)$. Thus, the original expansion is indeed a $\mathrm{KL}$ expansion.

$(\Rightarrow)$ To proceed, we need the following uniqueness conditions on (i) solution of (5a), and (ii) KL expansion of a random process.

Proposition 1 ( [30], Chap. 5): Given, the non-explosion condition and the Lipschitz condition are satisfied for $f(\cdot, \cdot)$ in (5a). Let $Z$ be a random variable, independent of the $\sigma$ algebra generated by $\eta(\omega, t), t \geq 0$, and $\mathbb{E}\left[|Z|^{2}\right]<\infty$. Then the SDE 5a where $t \in[0, T], X(\omega, 0)=Z$, has a unique $t$-continuous solution $x(\omega, t)$ adapted to the filtration $\mathscr{F}_{t}^{Z}$ generated by $Z$, and $\mathbb{E}\left[\int_{0}^{T}|x(\omega, t)|^{2} d t\right]<\infty$.

Proposition 2 ( [28], Chap. 2): The Karhunen-Loève expansion of a random process $x(\omega, t)$, given by $x(\omega, t)=$ $\sum_{i=1}^{\infty} \sqrt{\Lambda_{i}} \zeta_{i}(\omega) e_{i}(t)$, is unique.

Let us assume that $\breve{x}_{N}(\omega, t)$ is the KL expansion of $x(\omega, t)$. Furthermore, if possible, assume that $\breve{x}_{N}(\omega, t) \neq x_{N}(\omega, t)$, which is the solution of (6) and converges to the solution of (5a) in m.s. sense.

Notice that (6) has unique solution as RHS of (6) satisfies Lipschitz condition. This can be proved as follows: for RHS of (6) to satisfy Lipschitz condition, we must have $\left|f(x, t)+\eta_{N}(\omega, t)-f(\breve{x}, t)-\eta_{N}(\omega, t)\right| \leq C|x-\breve{x}|$, which is true since $f(\cdot, \cdot)$ itself satisfies Lipschitz condition. Hence (5a) has unique solution that admits a unique KL expansion. Also according to our assumption, the solution of (6) converges to the solution of 5a in m.s. sense. This contradicts our assumption that $\breve{x}_{N}(\omega, t) \neq x_{N}(\omega, t)$, which completes the proof.

\section{Proof for Theorem 2}

Integrating (8) and $(9)$ and taking the expected value of square of the difference, we obtain

$$
\begin{aligned}
& \mathbb{E}\left|x(t)-x_{N}(t)\right|^{2}=\mathbb{E}\left[\mid\left(x(0)-x_{N}(0)\right)+\int_{0}^{t}(f(x, s)-\right. \\
& \left.\left.f\left(x_{N}, s\right)\right) d s+\left.\int_{0}^{t} d\left(\mathcal{W}_{s}-\sum_{i=1}^{N} \sqrt{\Lambda_{i}} \zeta_{i}(\omega) e_{i}(s)\right)\right|^{2}\right] \\
& \leq \underbrace{\mathbb{E}\left|\left(x(0)-x_{N}(0)\right)\right|^{2}}_{0=: B \text { (say) }}+\mathbb{E}\left|\int_{0}^{t}\left(f(x, s)-f\left(x_{N}, s\right)\right) d s\right|^{2}+ \\
& \mathbb{E}\left|\int_{0}^{t} d\left(W_{s}-\sum_{i=1}^{N} \sqrt{\Lambda_{i}} \zeta_{i}(\omega) e_{i}(s)\right)\right|^{2}, \\
& \leq B+t \mathbb{E} \int_{0}^{t}\left|f(x, s)-f\left(x_{N}, s\right)\right|^{2} d s+ \\
& \mathbb{E}\left|\int_{0}^{t} d\left(W_{s}-\sum_{i=1}^{N} \sqrt{\Lambda_{i}} \zeta_{i}(\omega) e_{i}(s)\right)\right|^{2},
\end{aligned}
$$


where in the last step, we used Chebyshev's integral inequality. Consequently, we have

$$
\begin{aligned}
& \lim _{N \rightarrow \infty} \mathbb{E}\left|x(t)-x_{N}(t)\right|^{2} \leq B+\lim _{N \rightarrow \infty} t \mathbb{E}\left[\int_{0}^{t}\left|f(x, s)-f\left(x_{N}, s\right)\right|^{2}\right. \\
& d s]+\lim _{N \rightarrow \infty} \mathbb{E}\left|\int_{0}^{t} d\left(W_{s}-\sum_{i=1}^{N} \sqrt{\Lambda_{i}} \zeta_{i}(\omega) e_{i}(s)\right)\right|^{2}
\end{aligned}
$$

Using the Lipschitz criterion and property of KL expansion, from (23) we get

$$
\begin{aligned}
& \underbrace{\lim _{N \rightarrow \infty} \mathbb{E}\left|x(t)-x_{N}(t)\right|^{2}}_{v(t) \text { (say) }} \leq B+t C \int_{0}^{t} \lim _{N \rightarrow \infty} \mathbb{E}\left|x(s)-x_{N}(s)\right|^{2} d s \\
& \Rightarrow v(t) \leq B+A \int_{0}^{t} v(s) d s \Rightarrow v(t) \leq B \exp (A t)
\end{aligned}
$$

where the last step follows from Gronwall's inequality, with $t C \leq A, \forall t \in(0, T]$. Therefore, $\lim _{N \rightarrow \infty} \mathbb{E}\left|x(t)-x_{N}(t)\right|^{2}=$ 0 , since $x(0)=x_{N}(0) \Rightarrow B=0$, as per our assumption.

\section{Proof for Corollary 3}

In the proof of Theorem 2 for $x(0) \neq x_{N}(0)$, taking the limit $N \rightarrow \infty$ yields

$$
\begin{array}{r}
\lim _{N \rightarrow \infty} \mathbb{E}\left|x(t)-x_{N}(t)\right|^{2} \leq \lim _{N \rightarrow \infty} \mathbb{E}\left|\left(x(0)-x_{N}(0)\right)\right|^{2}+ \\
\lim _{N \rightarrow \infty} t \mathbb{E} \int_{0}^{t}\left|f(x, s)-f\left(x_{N}, s\right)\right|^{2} d s+ \\
\lim _{N \rightarrow \infty} \mathbb{E}\left|\int_{0}^{t} d\left(\mathcal{W}_{s}-\sum_{i=1}^{N} \sqrt{\Lambda_{i}} \zeta_{i}(\omega) e_{i}(s)\right)\right|^{2} .
\end{array}
$$

Going through the subsequent steps as before, we arrive at

$$
\lim _{N \rightarrow \infty} \mathbb{E}\left|x(t)-x_{N}(t)\right|^{2}=0 \text {, if } \lim _{N \rightarrow \infty} \mathbb{E}\left|x(0)-x_{N}(0)\right|^{2}=0 .
$$

However, if $x_{N}(0)$ is the gPC expansion of $x(0)$, then they asymptotically converge in m.s. sense [28]. Hence $\lim _{N \rightarrow \infty} \mathbb{E}\left|x(0)-x_{N}(0)\right|^{2}=0$, which, from the Gronwall's inequality, implies that $\lim _{N \rightarrow \infty} \mathbb{E}\left|x(t)-x_{N}(t)\right|^{2}=0$. This completes our proof.

\section{REFERENCES}

[1] M.S. Arulampalam, S. Maskell, N. Gordon, and T. Clapp, "A Tutorial On Particle Filters for Online Nonlinear/Non-Gaussian Bayesian Tracking", IEEE Transactions on Signal Processing, Vol. 50, No. 2, pp. 174-188, 2002.

[2] F. Daum, and J. Huang, "Curse of Dimensionality and Particle Filters", Proceedings of the 2003 IEEE Aerospace Conference, Vol. 4, pp. 1979-1993, 2003.

[3] A. Doucet, S. Godsill, and C. Andrieu, "On Sequential Monte Carlo Sampling Methods for Bayesian Filtering", Statistics and Computing, Vol. 10, No. 3, pp. 197-208, 2000.

[4] C.S. Manohar, and D. Roy, "Monte Carlo Filters for Identification of Nonlinear Structural Dynamical Systems", Sadhana, Vol. 31, No. 4, pp. 399-427, 2006.

[5] N.J. Gordon, D.J. Salmond, and A.F.M. Smith, "Novel Approach to Nonlinear/Non-Gaussian Bayesian State Estimation", IEE Proceedings of Radar and Signal Processing, Vol. 140, No. 2, pp. 107-113, 1993.

[6] B. Ristic, S. Arulampalam, and N. Gordon, Beyond the Kalman Filter: Particle Filters for Tracking Applications, Artech House Publishers, 2004.

[7] N. Oudjane, and C. Musso, "Progressive Correction for Regularized Particle Filters", Proceedings of the Third International Conference on Information Fusion, Vol. 2, pp. THB2-10, 2000.
[8] W.R. Gilks, and C. Berzuini, "Following A Moving Target-Monte Carlo Inference for Dynamic Bayesian Models", Journal of the Royal Statistical Society: Series B (Statistical Methodology), Vol. 63, No. 1, pp. 127-146, 2001.

[9] C. Snyder, T. Bengtsson, P. Bickel, and J. Anderson, "Obstacles to High-dimensional Particle filtering”, Monthly Weather Review, Vol. 136, No. 12, pp. 4629-4640, 2008.

[10] G. Casella, and C.P. Robert, "Rao-Blackwellisation of Sampling Schemes", Biometrika, Vol. 83, No. 1, pp. 81-94, 1996.

[11] A. Halder, and R. Bhattacharya, "Dispersion Analysis in Hypersonic Flight During Planetary Entry Using Stochastic Liouville Equation", Journal of Guidance Control and Dynamics, Vol. 34, No. 2, pp. 459474, 2011.

[12] A. Lasota, and M.C. Mackey, Chaos, Fractals, and Noise: Stochastic Aspects of Dynamics, Springer, Vol. 97, 1994.

[13] P. Dutta, and R. Bhattacharya, "Hypersonic State Estimation Using the Frobenius-Perron Operator", Journal of Guidance Control and Dynamics, Vol. 34, No. 2, pp. 325-344, 2011.

[14] H. Risken, The Fokker-Planck Equation: Methods of Solution and Applications, Springer, Vol. 18, 1996.

[15] R. Bellman, Dynamic Programming, Princeton University Press, 1957.

[16] P. Dutta, A. Halder, and R. Bhattacharya, "Uncertainty Quantification for Stochastic Nonlinear Systems using Perron-Frobenius Operator and Karhunen-Loéve Expansion", IEEE Multi-Conference on Systems and Control, Dubrovnik, Croatia, 2012.

[17] M. Grigoriu, "White Noise Processes", Journal of Engineering Mechanics, Vol. 113, No. 5, pp. 757-765, 1987.

[18] M. Grigoriu, Stochastic Calculus: Applications in Science and Engineering. First ed., Birkhäuser; 2002.

[19] C. Villani, Topics in Optimal Transportation. Graduate Studies in Mathematics, First ed., American Mathematical Society; 2003.

[20] A. Halder, and R. Bhattacharya, "Further Results on Probabilistic Model Validation in Wasserstein Metric", $51^{\text {st }}$ IEEE Conference on Decision and Control, Maui, 2012.

[21] C.R. Givens, and R.M. Shortt, "A Class of Wasserstein Metrics for Probability Distributions", The Michigan Mathematical Journal, Vol. 31, No. 2, pp. 231-240, 1984.

[22] A. Halder, and R. Bhattacharya, "Model Validation: A Probabilistic Formulation", 50 th IEEE Conference on Decision and Control, Orlando, 2011.

[23] F. Daum, "Exact Finite-dimensional Nonlinear Filters", IEEE Transactions on Automatic Control, Vol. 31, No. 7, pp. 616-622, 1986.

[24] V.E. Beneš, "Exact Finite-dimensional Filters for Certain Diffusions with Nonlinear Drift", Stochastics: An International Journal of Probability and Stochastic Processes, Vol. 5, No. 1-2, pp. 65-92, 1981.

[25] A. Bain, and D. Crişan, Fundamentals of Stochastic Filtering, Vol. 60, Springer Verlag, 2008.

[26] D. Crisan, "A Direct Computation of the Beneš Filter Conditional Density", Stochastics: An International Journal of Probability and Stochastic Processes, Vol. 55, No. 1-2, pp. 47-54, 1995.

[27] S. Chib, and E. Greenberg, "Understanding the Metropolis-Hastings Algorithm”, American Statistician, Vol. 49, No. 4, pp. 327-335, 1995.

[28] R.D. Ghanem, and P.D. Spanos, Stochastic Finite Elements: A Spectral Approach, Dover Publications, 2003.

[29] F. Daum, and M. Krichman, "Non-particle Filters", Proceedings of SPIE, Vol. 6236, 2006.

[30] B.K. Øksendal, Stochastic Differential Equations: An Introduction with Applications, Springer Verlag, 2003. 